\title{
Modeling prefrontal functions for robot navigation
}

\author{
Hervé Frezza-Buet, frezza@loria.fr and Frédéric Alexandre, falex@loria.fr, \\ LORIA, BP 239, 54506 Vandoeuvre Cedex, France
}

\begin{abstract}
This paper presents a model of cooperation between representations of correlation in the associative cortex and goal oriented representations of action in the prefrontal cortex. The model is applied to robot navigation and gives a framework for addressing planning with numerical neural techniques.
\end{abstract}

\section{Introduction}

Neural networks have turned out to be suitable for numerical problems, as supervised and unsupervised classification of numerical vectors. This capability of neural techniques to deal with numerical data is required when addressing autonomous behavior of a real robot, because perceptions from sensors are numerical and noisy. But behavior is much more than classifying perceptions. Behaving implies dealing with constraints, like keeping internal variables within vital range by exploiting resources of the external world or avoiding obstacles. Taking these constraints into account leads the robot to plan actions. As opposed to computation of numerical data, only few attempts have been made to use neural techniques for planning, because planning seems to be a symbolic task.

The purpose of this paper is to show that recent works from biology concerning prefrontal cortex modeling allow to design neural control architectures for robot behavior that include planning capabilities. As symbols are often difficult to ground on numerical data, the challenge of planning with neural networks is to avoid this grounding problem by keeping computation at a numerical level and making planning emerge from interaction of neurons.

The task we use to design our model is a simulated application for the moment, and the model is to be tested on a real robot. The experimental framework related to the work presented in this paper is a simulated robot in a flat environment. Motions are continuous and colored patches on the floor corresponds to food areas, water areas and obstacles. The robot perceives the environment through a 360 degrees video camera. It has to survive by "drinking" on water areas and "eating" on food areas.
The architecture of the robot consists of two parts : a model of associative cortex and a model of prefrontal cortex. The cortical framework and the respective roles of the two models will be first presented, and the cortical associative model we use will be described. Then we will present the main features of our model of prefrontal cortex and conclude with a discussion of experimental results.

\section{The cortical framework}

The cortex has been described as a bi-dimensional sheet tiled with replicated neural circuits: the cortical columns [4]. Cortical columns receive inputs from the external world, from other columns of the cortex and from other parts of the brain. As the cortical column is a large set of neurons, it is successfully modeled by an automaton [1], dealing with input/output signals through several states of activation. That is why the units of our model are far from the formal neuron described in [16] and used in perceptrons.

Within our model, each unit is associated to a perceptive or motor event. Units that concern events of the same modality are gathered within cortical maps. Primary maps then gather mono-modal units, related to sensors or effectors, and associative maps, owning units connected to units of other cortical maps, allow to represent multi-modal events. The biological basis for cortical maps and the way they are connected can be found in [2], but our architecture only deals with associative maps linking two other maps.

The functioning of the units is inspired from biological observations [4]: A unit has three kinds of activity. First, it can be at rest, because of absence of the external event it is tuned on, or because of inhibition from other units. Second, the unit can be excited, when the associated external event occurs. Third, the unit can be called, the call activity representing the fact that the cortex, for any reason, needs for the occurrence of the event associated to the called unit. A called unit can thus be viewed as a goal.

It has been observed in the cortex that learning consists in specializing some columns, among a set of 
columns having the same connections [4]. A convenient way to model this learning through specialization is to first use a single unit, and then allow it to split when something has to be learned [9]. Our cortical model is then incremental.

\section{The respective role of posterior and prefrontal cortex models}

\section{A. Why making that distinction?}

Some cortical models addressing behavior can be found in the literature, and some of them provide behavioral capabilities with only one kind of cortical unit [3], [8]. On the one hand, the unit in such model have to deal with perceptions, detecting causal relationships between perceptive events in order to learn rules of the external world. On the other hand, the system has to take current needs into account, i.e. build sequences of goals and subgoals that control behavior for goal reaching. This sequence management can be global [3], or at the level of each unit [8]. As sequences of perceptions are often noisy, an efficient management of sequences tends to be complex, and a large computational part of the cortical units is devoted to this goal management, whereas the cortical unit is supposed to represent a perceptive event.

The idea is then to separate perception-related computation from goal-related computation. This separation is inspired from biological findings concerning the organization of the cortex [10]: whereas the posterior (associative) cortex is the perceptive pole of the cortex, the prefrontal cortex appears to be dedicated to goal-oriented representation.

\section{B. What can we expect from a model of associative cor- tex?}

As we make a distinction between associative cortex computation and frontal cortex computation within our model, we try to define the limits of the role of associative cortex. Our model of associative cortex is designed for correlation detection between multi-modal perceptions. These correlations can be both spatial and temporal. Time management is sometimes reported as a specificity of prefrontal cortex. On the contrary, we actually consider computation of temporal correlations within associative cortex. These correlations are short-term associations between consecutive events of the world, and they can be used as some kind of one step ahead prediction. Associative cortex representations, within our model, are the result of the learning of the transition function between states of the perceptive world, i.e. they represent the regularity of the world as a kind of differential equation.

For example, hand controling with vision during a reaching task requires to learn associations of the position of the hand on the retina with motor commands to muscles [5]. These associations allow to predict immediate consequences of actions, i.e choosing the correct muscle command to make the hand be in the desired place on the retina.

Using the associative model then consists in calling a desired perceptive event (i.e. creating a call activity within the corresponding cortical column). This call will propagate to columns corresponding to close events. If one of these latter columns corresponds to a current event, the associative cortex triggers the action that allows to get the initially desired perceptive event. This principle is illustrated in section IV.

\section{Computing behavior with prefrontal cortex model}

We roughly consider the role of the prefrontal cortex model as controling sequences of calls toward associative cortex. Each of these calls aims at triggering an action to attain an elementary perceptive state from the current one (the latter being relatively close to the former). Suitable sequences of calls are those who lead to a behavior that maximizes reward, making a compromise between multiple vital needs and unexpected features of the world.

1) Functional view: We present in this section some of the main features of prefrontal cortex computation, according to functional descriptions found in the biological literature. These results are mainly obtained by studies with impaired patients that are not presented in this paper.

- Prefrontal cortex is strongly connected to limbic structures dealing with drives and rewards. These connections "[impart] prefrontal functions their eminently "active" character" [10]. As opposed to associative cortex that learn transition function of the world, prefrontal cortex units are driven by some kind of reinforcement learning rules.

- Prefrontal cortex, according to observations during delayed-response task experiments, can play the role of a "working memory" [12], that stores current context when this context is relevant for getting a reward. A delayed-response task consists in presenting a cue, removing the cue during a delay period, and then presenting a situation to the subject. The way to get rewarded depends on the initial cue, and a subject that succeeds in getting the reward has to keep the cue in mind during the delay period. Frontal cortex plays a central role in such capabilities [12], and this role have been 
modeled in [7]. Models and observations involve neurons that have a sustained activity during the delay period.

- As mentioned in [10], the prefrontal cortex provides the ability to bind time separated events in order to build temporal gestalts. This binding role is the result of the processing of "cross-temporal contingencies". Such representations enable to anticipate long term consequences of actions, and can be viewed a a "memory of future" [10]. "Set cells" have been observed that fire before the occurrence of an event. The anticipatory function of those cells is increasing the sensibility of associated cortical cells, that can be interpreted as a call in the associative cortex, according to the definition of call activity in [4].

- The planning ability of the prefrontal cortex is grounded on its ability to schedule contention [17]. A large part of prefrontal activity consists of inhibiting influences of other possible sequences of action. This allows to keep attention to the current plan, without being distractible.

- Last, let us mention a central function of frontal activity. This function concerns the ability to quickly adapt to unexpected events. An expected reward that actually cannot be obtained will result in a fast changing of the current strategy. This property has been modeled in [7].

2) Biologically plausible bistable mechanism : A biologically plausible mechanism has been proposed in [4], and it has been used for modeling experimental data in go-no go tasks [13]. The idea is to consider the sustained activity observed with frontal neurons as a bistable behavior of the prefrontal unit. Each frontal unit is associated with a perceptive event (i.e. a unit in the associative cortex) and is able to store a call on this event if the associative cortex is not able to get the event (i.e. there is no direct action to trigger to get the desired perceptive state). This storing provide stacking capabilities, as described in the following example (figure 1).

Let us suppose that getting events $A_{1}$ and $A_{2}$ is required for being rewarded. Moreover, let us suppose that getting successively $A_{11}, A_{12}$ and $A_{13}$ is required to make a call in $A_{1}$ efficient enough to get $A_{1}$. In the same way, let us suppose that getting $A_{12}$ requires getting $A_{121}$ and $A_{122}$. The behavior of the frontal associated bistables $F_{i}$ is the following. First, $F_{1}$ is called. A transient call is propagated to $A_{1}$ without success in getting $A_{1}$. Then $F_{1}$ goes to the ON state and calls $F_{11}$. The oN state of $F_{1}$ is an uneffective call in $F_{1}$, that only

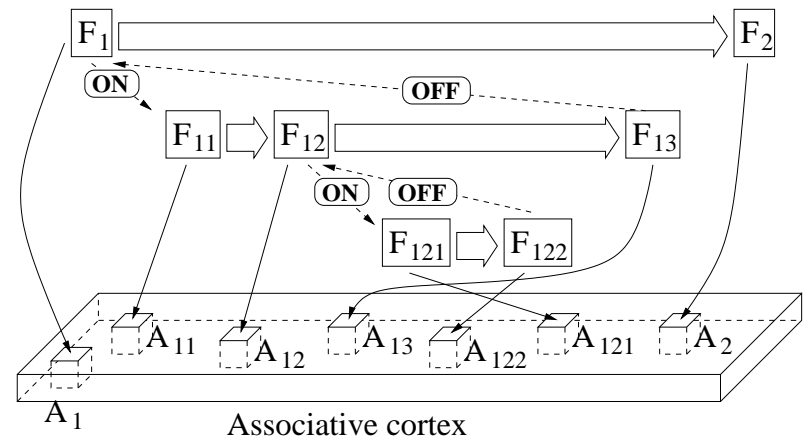

Fig. 1. Hierarchical organization of bistables: stacking property of prefrontal units.

aims at stacking the initial goal of calling $A_{1}$. In the same way, $F_{11}$ calls $A_{11}$. When $A_{11}$ is excited (the call has succeeded), the call in $F_{11}$ spreads to $F_{12}$. As for $F_{1}, F_{12}$ goes to the on state, stacking the subgoal of calling $A_{12}$ and call is transmitted to $F_{121}$, and then to $A_{121}$. When $A_{121}$ occurs, call is transmitted to $F_{121}$, and then to $A_{122}$. When $A_{122}$ occurs, $F_{122}$ makes $F_{12}$ go to the transient OFF state. The calling of $A_{12}$ during the on state is made effective on $A_{12}$. When $A_{12}$ is obtained, call is transmitted to $F_{13}$ : the call previously stored in $F_{12}$ is popped up from the stack. In the same way, call will be transmitted to $A_{2}$ after $A_{13}$ and $A_{1}$ are obtained, popping up the call initially stored in $F_{1}$.

On the basis of this stacking mechanism, sustained activities observed in frontal neurons have to be related to the ON state of units in the model. This storage can be viewed as a prediction of the OFF state (memory of the future, cross-temporal contingencies), or as a context for further computations (working memory).

Last, if a call that is expected is not obtained, the local "frustration" may trigger the stacking of that call while trying to get it by using other sequences. If this latter sequence succeeds in allowing the success of the stored call, it will be introduced by learning in the hierarchical structure of bistables. This can be related to the capacity of the prefrontal cortex to quickly adapt to unexpected events.

\section{The associative cortex model}

The associative cortex model consists in several maps connected to perceptions and actions. Perceptions of the robot are the video input and the state of internal vital variables, and actions to the world are eating, drinking, turning (with a continuous angle) and going ahead. Another set of actions is defined, concerning ocular saccades. Primary maps are connected to actuators or sensors, whereas associative maps combine primary maps. The global architecture is sketched in figure 2 and detailed below. 


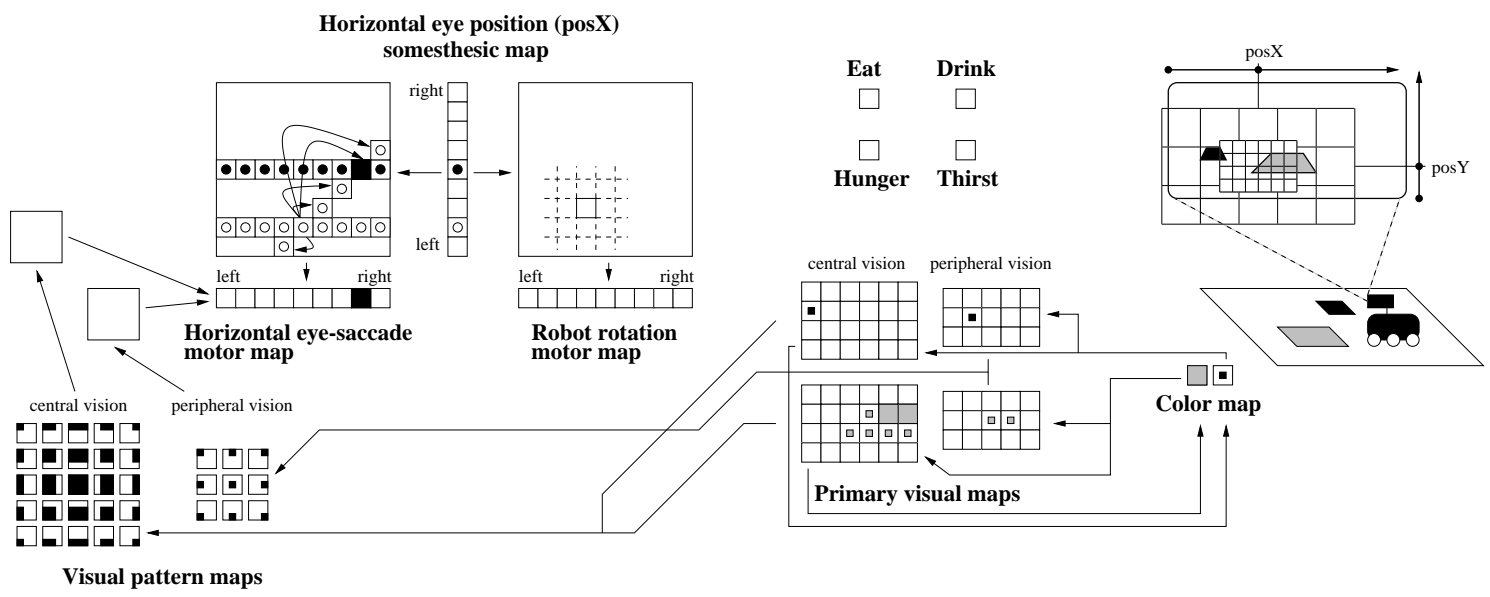

Fig. 2. Architecture of the model of associative cortex.

\section{A. Ingestions maps}

These four maps are very straightforward in our model, they all contain one unit. The "Eat" and "Drink" maps contain units that trigger the corresponding action when excited. The "Hunger" and "Thirst" maps are somesthesic maps, whose units perceives the global sensation of being "hungry" and "thirsty" (the corresponding unit is excited).

\section{B. Motor and saccades maps}

Motor maps contain units that trigger a motion when excited. The "Rotation" map owns units that are topologically organized, from the triggering of a strong left rotation to the triggering of a strong right rotation. If several rotation units are excited, the mean rotation angle is performed by the robot, units in the map acting as a population vector [11]. A "go-ahead' map, not represented on figure 2 , is organized the same way, from low speed to high speed motions. Two other "Horizontal eye-saccade" and "vertical-eye saccade" (not represented on figure) are used to move an attention point in the video image.

\section{Attention point maps}

These two maps (only "horizontal eye position" is in figure 2) are perceptive maps whose units are excited for a particular abscissa (resp. ordinate) of the attention point. The robot then perceives the location of the point it stares in the picture, which is a self-centered referential.

\section{Visual maps}

Two virtual overlapping grids are centered on the position (pos $X, p o s Y)$ of the attention point (see fig- ure 2), respectively corresponding to central and peripheral vision. There are two visual maps for each color to be detected, one concerning the central vision and one concerning peripheral vision. If one unit of a "central vision" map is excited (the corresponding color is perceived on the corresponding place in the picture), the corresponding unit in the "color map" is excited. Reversely, a call activity in a unit in the "color map" make all units in the two corresponding central and peripheral visual maps increase the intensity of their response (priming effect of call).

Then, calling a color unit allows the corresponding primary visual maps to own columns that are excited. This excitation is then strong enough to make the units of the "visual patterns" maps be excited. The excited patterns are those who fit best the activation distribution in the primed primary visual maps. The "visual patterns" maps are topologically organized, as observed in [14] and modeled with SOM architecture [15].

\section{E. Associative maps}

In our model, an associative map combines two perceptive and motor maps. Its role is to use motor commands to make call units in perceptive maps be excited, i.e. to allow to get the desired perceptive event by triggering the appropriate action.

An example is detailed for the positioning of the attention point to a desired place in the picture. Let us suppose that the desired abscissa of the attention point is "near extreme left", the corresponding unit of the "horizontal eye position is called" (empty circle in figure 2). If the actual position of the attention point is "centered" (black circle), the call will spread in the associative map to directly associate units (see the row of empty circles), and from there it will spread to other units in the associative map through learned links (ar- 
rows on the figure). This priming call then allows some units (one in the example) to be excited enough to make the corresponding motor unit trigger the appropriate saccade. The same is done (but is not represented) for vertical positions and saccades.

The topological organization of visual patterns allows to do the same with abscissa and ordinate of the pattern in the map. If a pattern unit is called and if another is perceived, the saccade that will be triggered through an appropriate associative map will move the attention point so that the desired pattern is close to the attention point. It allows to look for color patches with peripheral vision (call in the "centered" pattern of peripheral vision), and then to stare an edge, an upper left angle, etc. with patterns of central vision.

Concerning motion, the principle is the same. First, a color unit and a pattern unit have to be called, so that the ordinate of the attention point corresponds to the target in the picture. Then, associative maps allow to turn (see "Robot rotation" map) to make the target centered (call on center unit in "Horizontal eye position"). Reaching the targeted area is provided the same way by associating vertical position of the attention point with "go ahead" map (not on figure 2).

\section{F. Using the model}

The use of associative cortex is then robust and quite simple. For example, if the robot has to reach a blue area, the unit in the "color map" corresponding to blue has to be called. Then a call on the unit of the peripheral vision pattern map corresponding to pattern - will make the attention point fix a blue patch. Then the latter call has to be stopped, and a call in the center vision pattern map corresponding to pattern $\boldsymbol{\square}$ has to be sustained. A call in bottom center position of attention point will make the robot center the target and reach it, by turning and going ahead until the called pattern is at the bottom center of the picture.

The model can also be used to avoid obstacles. If obstacles are grey, an analog call sequence, for grey color unit and central vision pattern $\square$, make the robot turning around the grey area, with this area on its left.

\section{The prefrontal cortex model}

\section{A. Architecture of the model}

The architecture of the model is more basic than the hierarchical organization of the associative cortex. The prefrontal cortex model consists of one map gathering prefrontal units. Each of these units is connected to a unit in the associative cortex. A prefrontal unit sends calls to the corresponding associative unit and receives excitation from it when the perceptive event occurs. In order to reduce the number of frontal units to manage, we only choose a subset of associative units to be connected to a frontal unit (those in the color map, the central pattern of peripheral vision, left-bottom, center-bottom and right bottom central vision patterns, the units of the four ingestion maps, and those of the "posX" and "posY" map). Units in the frontal map are totally interconnected, and they can split when specializing for a particular computation after learning. The learning rules, not detailed in this paper, are temporal correlation rules.

The drive is a global scalar parameter that is high when one of the vital variables is weak, and reward occurs when the level of one vital variable increases. reward is also a global scalar parameter.

\section{B. Functioning of units}

As we mentioned, each frontal unit is connected to an associative unit. The frontal unit is "activated" when the frontal unit is excited. When the end of activation of a unit is correlated with the global reward, the unit is said to be a "need unit". Mainly, units that correspond to the sensitive effects of drives on the "body" are need units, because the sensation stops when reward occurs. In our model, "hunger" and "thirst" frontal units are considered, after learning, as need units. Need units play the role of perceptions from the body that are related to motivational state. This has been inspired from the somatic markers described in [6].

The stronger the global drive is, the more frequently frontal units randomly start to trigger a call on the associative cortical unit. This "calling" state has a random duration. This allows to try some patterns of calls on the associative cortex. If a calling unit is excited, it is said to be "successful". This latter state is more related to action than the activated state which depends only on perceptions. The learned probabilities of activations of other units when a unit is successful defines the "perceptive context" of the unit. The perceptive context is used to increase the frequency of the random trigger of call.

A need unit, whose decrease of activation is related to reward, can learn correlations between this decrease and success of other units in the map. These correlations define the "effective context". It has to be viewed as the actions that actually work when the reward occurs. When a need unit is active and "knows" its effective context (it has split), the units of that context have a high probability to trigger a sustained call on the corresponding associative unit.

The bistable activity is then grounded on the following principle. When a drive unit $D$ has computed is ef- 
fective context, the success of the units of that context is an action that is supposed to lead to the reward of the drive unit. If not, because of a change in the external world, the units $C_{i}$ in the effective context go to the ON state. Units in the oN state behave as drive units: they allow spontaneous calls on other units in a map in order to compute their own effective context (the $C_{i}$ splits when it is learned). When this latter context is learned, the specialized unit $C_{i}^{\prime}$ is a drive unit, being activated when $D$ is activated, but rewarded when its specific effective context is obtained.

To sum up, the initial task is to solve the drive $D$. When a solution $C_{i}$ is found, it is used until the external world changes. The reaction of this change is first a random try of other calls on the associative units (see random behavior of rule units in [7]), until an efficient solution $C_{i}^{\prime}$ is found. Then, bistable activities of $D$ and $C_{i}^{\prime}$ are analog to those described in section 2).

\section{Experiments}

Experiments are based on step by step changes in the external world, as it is the case in the learning of delayed-response tasks [13]. First, the robot is set on the area corresponding to its current need, so that it learns that "hunger" is a drive unit whose effective context is "eat", and the same for "thirst" and "drink". Then, when learning is performed, i.e. when the robot eat and drink when it is respectively hungry and thirsty, we randomly put the robot on a wrong area, so that the effective context for drinking is "asking for a blue color at the bottom center", and the same for eating. Then, the changing consists in putting the robot near the area, etc.

Currently, convergence is quite long, and inhibition mechanisms between calls that can be simultaneously tested are still complex and not robust enough. Nevertheless, the model gives a framework for making planning capabilities emerge from a set of units, dealing with different kinds of needs, with a unified drive and reward management. Moreover, the architecture works on-line, not requiring start signals or discrete step by step events where consequences of action at time $t$ must occur at time $t+1$.

\section{Discussion}

Encouraging results observed with the model leads ongoing works to focus on a refinement of inhibition mechanism between frontal units allowed to call on the one hand, and a use of the associative cortex by the prefrontal cortex to predict consequences of calls on the other hand, in order to help search of adapted calls when the world changes. This will make the model quicker to find effective context of drive units.

\section{References}

[1] F. Alexandre, F. Guyot, J.-P. Haton, and Y. Burnod. The Cortical Column: A New Processing Unit for Multilayered Networks. Neural Networks, 4:15-25, 1991.

[2] D. H. Ballard. Cortical connections and parallel processing : Structure and function. The Behavioral and Brain Sciences, 9:67-129, 1986.

[3] P. Blanchet. An Architecture for Representing and Learning Behaviors by Trial and Error. In Proceedings Congress SAB'94: from Animals to Animats III, Simulation of Adaptative Behavior, Brighton (England), August 1994.

[4] Y. Burnod. An adaptive neural network : the cerebral cortex. Masson, 1989.

[5] Y. Burnod, P. Grandguillaume, I. Otto, S. Ferraina, P. Johnson, and R. Caminiti. Visuo-motor transformations underlying arm movements toward visual targets: a neural network model of cerebral cortical operations. In $\mathrm{J}$. Neurosc., volume 12, pages 1435-1453, 1992.

[6] A. R. Damasio. On some functions of the human prefrontal cortex. In J. Grafman, K. J. Holyoak, and F. Boller, editors, Structure and Functions of the Human Prefrontal Cortex, volume 769, pages 241-251. Annals of the New York Academy of Sciences, 1995.

[7] S. Dehaene and J.-P. Changeux. A simple model of prefrontal cortex function in delayed-response task. Journal of Cognitive Neuroscience, 1(3):244-261, 1989.

[8] H. Frezza-Buet and F. Alexandre. Selection of action with a cortically-inspired model. In Seventh European Workshop on Learning Robots, pages 13-21, 1998.

[9] H. Frezza-Buet and F. Alexandre. Specialization within cortical models: An application to causality learning. In Proceedings of the 7th European Symposium on Artificial Neural Networks, 1999.

[10] J. M. Fuster. The Prefrontal Cortex, Anatomy, Physiology, and Neurophysiology of the Frontal Lobe. Raven Press, New York, seconde edition, 1989.

[11] A. P. Georgopoulos, J. T. Lurito, M. Petrides, A. B. Schwartz, and J. T. Massey. Mental rotation of neuronal population vector. Science, 243:234-236, 1989.

[12] P. S. Goldman-Rakic. Modulation of memory fields by dopamine $\mathrm{d} 1$ receptors in prefrontal cortex. Nature, 376:572-575, 1995.

[13] E. Guigon, B. Dorizzi, Y. Burnod, and W. Schultz. Neural correlates of learning in the prefrontal cortex of the monkey: A predictive model. Cerebral Cortex, 5(2):135-147, 1995.

[14] D. H. Hubel and T. N. Wiesel. Functional architecture of macaque monkey visual cortex. Ferrier Lecture Proc. Roy. Soc. London, pages 1-59, 1977.

[15] T. Kohonen. Self-Organization and Associative Memory. Springer-Verlag, 1988.

[16] F. Rosenblatt. The perceptron: a probabilistic model for information storage and organization in the brain. In J. A. Anderson and E. Rosenfeld, editors, Neurocomputing: Fundations of Research (1989), pages 89-92. The MIT Press, 1958.

[17] D. T. Stuss, T. Shallice, M. P. Alexander, and T. W. Picton. A multidisciplinaty approach to anterior attentional functions. In J. Grafman, K. J. Holyoak, and F. Boller, editors, Structure and Functions of the Human Prefrontal Cortex, volume 769, pages 191-211. Annals of the New York Academy of Sciences, 1995. 